\title{
Anticoagulant Therapy In Ischemic Stroke Or TIA
}

\author{
Dr Kaveh Mehrvar,M .D. ${ }^{1,2}$ \\ ${ }^{1}$ Neurology Ward, AAlinasab hospital,Tabriz, Iran. \\ ${ }^{2}$ Neurosciences Research Center(NSRC),Tabriz University of M edical Sciences, Iran. \\ *Corresponding Author: Email: kaveh_mehrvar@yahoo.com
}

Stroke is the leading cause of disability and the third leading cause of death. Anticoagulants have been used to treat patients with acute ischemic stroke for many years. Despite their widespread use, the usefulness of emergency anticoagulation is a subject of debate. Disagreements exist about the best agent to administer, the route of administration, the use of a bolus dose to start treatment, the level of anticoagulation required, and the duration of treatment. There are 2 types of anticoagulants: Parenteral and oral. Heparin is an anticoagulant that used parenteral. Oral anticoagulants are including Warfarin and new anticoagulants such as Dabigatrn,Rivaroxaban ,Apixaban and other newer drugs.

In patients with noncardioembolic ischemic stroke or TIA antiplatelet agents are treatment of choice and preferred to anticoagulants.

In cardioembolic ischemic stroke or TIA with high risk of reembolization anticoagulants are considered as preferred treatment. Warfarin, apixaban $10 \mathrm{mg} / \mathrm{d}$, Rivaroxaban20mg/d, and dabigatran $150 \mathrm{mg} / \mathrm{d}$ are all indicated for the prevention of recurrent stroke in patients with nonvalvular AF, whether paroxysmal or permanent.Also anticoagulant therapy is recommended for ischemic stroke or TIA patients in the setting of acute MI, atrial or ventricular thrombosis or dilated and restricted cardiomyopathy. Some valvular heart diseases are other indication for anticoagulant therapy in ischemic stroke or TIA patients.

Ischemic Stroke or TIA in patients with Cerebral vein thrombosis and known hypercoagulable state specially anti phospholipid antibody syndrome are other indications for anticoagulant treatment.

Key words: Ischemic Stroke, TIA, Anticoagulant therapy

DOI: $10.7575 /$ aiac.abcmed.ca1.60

Published Date: February 2017

Peer-review is under responsibility of the 9th Iranian Stroke Congress.

Published by Australian International Academic Centre, Australia

This published work is open access under the CC BY license.

Available online at www.abcmed.aiac.org.au 